\title{
La escuela cubana como contexto para el correcto desarrollo de la percepción ambiental
}

\author{
The Cuban school as a context for a proper \\ development of the environmental perception
}

\author{
Olga Natalia TSEREJ VÁZQUEZ ${ }^{1}$ y María Milagros FEBLES ELEJALDE ${ }^{2}$ \\ ${ }^{1}$ Museo Nacional de Historia Natural de Cuba \\ ${ }^{2}$ Facultad de Psicología de la Universidad de La Habana
}

Recibido: Mayo 2013

Aceptado: Noviembre 2013

\section{Resumen}

En Cuba la Psicología Ambiental es una disciplina nueva que promueve una visión histórica y cultural de los seres humanos. La percepción es uno de los procesos definidos tendientes a la conformación de una consciencia ambiental. En dependencia de la percepción del ambiente se desarrolla la acción e interacción con su entorno. Es decir que con una adecuada interacción también se desarrolla una buena percepción de los elementos significativos del ambiente y se contribuye a la aparición de la consciencia ambiental, de la cual, la percepción es uno de los principales procesos. En esta importante transformación la escuela es una de las principales vías de formación de conocimientos, habilidades, hábitos y actitudes frente al entorno. Es por esto que el diagnóstico del grado de desarrollo de la percepción ambiental en estudiantes permite detectar las debilidades que poseen los programas de estudio en cuanto a dimensión ambiental y contribuye a proponer soluciones sobre la base de problemas concretos. Este estudio se apoya en diferentes investigaciones realizadas a estudiantes cubanos provenientes de diferentes niveles de enseñanza y provee un primer acercamiento a la dinámica del desarrollo de la percepción del ambiente en estos individuos. En las últimas investigaciones se utilizaron como indicadores de desarrollo la representación de determinadas dimensiones del concepto de medio ambiente como son: la material o artefactual, relacional, intrapersonal, comportamental, cognoscitiva, natural o ecológica y cultural. En sentido general, se reflejan diferentes investigaciones que demuestran que la escuela es el contexto idóneo para el trabajo de educación ambiental.

Palabras clave: Educación ambiental, percepción ambiental, escuela, enseñanza

\begin{abstract}
Environmental Psychology in Cuba is a new discipline that promotes a historical and cultural vision of mankind. Perception is one of the distinct processes that creates environmental consciousness. Depending on the perception of the environment, individuals interact with it, and vice versa. It means that a good perception of the significant elements of the environment also contributes to the formation of an environmental consciousness, in which perception is one of the main processes. In this transformation the school is one of the most important places for
\end{abstract}


creating knowledge, skills, habits, and good attitudes towards the environment. As a result, the evaluation of the environmental perception development in students allows detecting weaknesses in the environmental education and proposing solutions based on specific problems. This study is based on different researches where the subjects were Cuban students from different educational levels and provides a first approach to the dynamic of the environmental perception development in these individuals. Recent researches have used some dimensions of the environment concept as development indicators: material, relational, intrapersonal, behavioural, cognitive, natural or ecological, and cultural. Generally speaking, different investigations show that school is the right context for environmental education.

Keywords: Environmental education, environmental perception, school, education

Los jóvenes de la época actual conviven en un planeta que cada vez más se encuentra afectado por diferentes tipos de degradación ambiental en una escala sin precedentes. Este deterioro ha resultado de la relación que hemos creado los seres humanos con la naturaleza (Anderson, 2007; Febles, 2011).

Como ha venido ocurriendo en todas las secuencias históricas, la sociedad evoluciona y esta evolución requiere cada vez más la utilización de recursos del entorno. En su implacable deseo de desarrollo, el ser humano ha destruido recursos insustituibles que no volverán a ser generados por la naturaleza, ha degradado elementos naturales indispensables para nuestra vida en la Tierra y está próximo a agotar nuestras fuentes de energía. De este modo el entorno en que vivimos se encuentra sometido a tantas presiones que exige cambios drásticos en las relaciones que la especie humana mantiene hacia él (Benegas y Marcén, 1995). Desde un punto de vista eco-psicológico el ser humano se encuentra como parte indisoluble del medio que lo rodea y su bienestar está estrechamente relacionado con el entorno en el cual se desarrolla (Stevens, 2010).

En el transcurso del proceso socio-histórico, el ser humano ha desarrollado su capacidad de alterar el ambiente, de forma inconsciente o premeditada (Martínez, 2012). Esta problemática se ha convertido en una cuestión ineludible desde la perspectiva de la psicología ambiental (Amérigo, 2006), la cual nos brinda herramientas poderosas para revertir el impacto que el ser humano está provocando en el ambiente. En este sentido es necesario explorar en las causas de este comportamiento inadecuado y particularmente en la formación de la esfera moral de los educandos. El trabajo ambiental promueve valores y nuevos motivos, en tanto promueve una mirada diferente hacia la naturaleza y la sociedad. El sistema de valores que posee un individuo lo ubica en un lugar en relación con la naturaleza. El desarrollo de estos valores ambientales es posible desde las más tempranas edades y es principalmente un proceso social que se va forjando progresivamente en las personas. Esto significa que los niveles de razonamiento moral se construyen y pasan por etapas diferentes. No cabe duda que la escuela reúne unas características esenciales que la hacen, en principio, idónea para proyectos de socialización o moralización de la población infantil y juvenil (Benegas y Marcén, 1995). Se ha demostrado que sujetos con estudios superiores muestran más actitudes pro-ambientalistas que los sujetos sin estudios (González y Amérigo, 1999). La escuela no sólo puede, sino que debe 
desencadenar un cambio actitudinal en los sujetos para favorecer su crecimiento moral; sólo así se podrá cambiar una sociedad (Yus, 1994). Se debe tener en cuenta que es en las escuelas donde se experimentan las primeras vivencias ambientales ya sea en asignaturas del nivel primario como "El mundo en que vivimos" (asignatura que se imparte en la enseñanza primaria y versa sobre fenómenos de la naturaleza y la sociedad) o en algún proyecto ambiental que en ellas se desarrolle. Las vivencias en sentido general son muy importantes pues regulan la dirección de nuestro desarrollo y a través de ellas se pueden producir efectos positivos emocionales y por ende, en nuestra percepción del ambiente. El espacio y las experiencias que provoca no son procesos aislados, sino que se relacionan con otros procesos sociales, formando parte de una lógica común de relación que permite a la convivencia de las personas con el espacio (Muñoz, 2007).

Cabe destacar que muchos de los problemas ambientales que hoy existen están dados porque las personas son incapaces de percibir su magnitud, es necesario informarles, guiarles; $\mathrm{y}$, cuándo y donde mejor que desde y en la escuela primaria. Allí se expresan las principales vivencias y se forman sus primeras percepciones del ambiente. La percepción es un proceso psicológico humano, una función del sistema psicológico en desarrollo que necesita actualizarse y que como proceso tiene un inicio y un fin, es una secuencia de momentos que se caracteriza por su enriquecimiento. Este enriquecimiento se alcanza como resultado de la actividad y de otros procesos dirigidos por la relación interpersonal, como lo es la educación que es portadora de conocimientos, vivencias y reflexiones en las personas lo que contribuye a su crecimiento (Alea, 2005). Si bien es cierto que existe el fenómeno de la constancia perceptual y sin ella sería difícil la vivencia y las conductas eficaces, la imagen perceptual sufre cambios en el tiempo, al igual que los sufre el ambiente que se percibe.

"El ambiente desde el punto de vista del psicólogo es un ambiente percibido por un sujeto, y el problema científico que atañe al psicólogo es averiguar cómo se produce ese precepto ambiental y cómo y en qué cuantía el ambiente modifica la conducta de los organismos que estudia" (Rodríguez, 1986). El ambiente, el espacio en que están ubicados los objetos de la realidad que es percibido por un sujeto, es personal y con significado único, de ello atestiguan la literatura psiquiátrica-existencial, las brillantes especulaciones de los idealistas subjetivos, el trabajo de artistas, críticos, arquitectos, pintores, escultores y antropólogos y es que el campo espacial está cargado de significados diferentes de un individuo a otro (Alea, 2005)

Es cierto que: "El análisis perceptivo clásico practicado por la psicología académica hasta 1960 no nos dice demasiado acerca de la percepción global del ambiente, aunque todo el estudio de los indicios y factores que intervienen en la percepción de la forma, el espacio y el movimiento, así como las influencias motivacionales y culturales sobre la percepción constituyen una base de conocimiento imprescindible para el ambientalista" (Rodríguez, 1986). Es de destacar que los estudios en Psicología Ambiental han estado enfocados desde una posición generalmente conductista en la que el ambiente se secciona en estímulos y no en un contexto social e histórico cultural que se significa por los sujetos en dependencia de 
sus vivencias, historias personales, familiares y comunitarias. Se hace imprescindible para la nueva Psicología Ambiental que se construya, partir del carácter cualitativo de los ambientes, independientemente de que en ellos se tengan que realizar estudios con una tecnología de avanzada adecuada, que brinden medidas objetivas, válidas y fiables de toda una serie de indicadores de calidad. En ella se deberá recalcar sobre el carácter aprendido y cambiante de la percepción y su dependencia de la actividad. Las percepciones ambientales han sido estudiadas en tanto se traducen en juicios que después orientan el comportamiento, así observamos los estudios de percepción de la calidad ambiental, la percepción del riesgo ambiental y la percepción estética del ambiente. En los estudios de la percepción de la calidad ambiental encontramos que el desarrollo perceptual determina una subjetividad que en forma de evaluaciones interviene en la elaboración de sus índices. Estos son importantes para ver la efectividad de su calidad y tomar decisiones ambientales. Se trata de índices de polución, calidad de residuos, calidad de paisajes o escenografía, etc. Ellos son importantes para evaluar resultados de programa, el impacto que puedan producir los cambios ambientales, las tendencias de preferencias para el público, etc. Una buena percepción de los elementos significativos del ambiente (alto nivel de desarrollo de la percepción), llámense de los problemas que lo afectan o de sus numerosas dimensiones, contribuye al desarrollo de la consciencia ambiental y por ende a una mayor protección y cuidado en las interacciones con él. Es por esto que conocer la variedad y nivel de desarrollo de las percepciones del ambiente que poseen las personas, en las diferentes etapas de su formación, ayudaría en la planificación de programas de educación ambiental orientado a fortalecer las debilidades detectadas a cada nivel.

En Cuba, la Psicología ambiental con su desarrollo incipiente ha desarrollado investigaciones que se relacionan en lo fundamental con la escuela como contexto de formación, sin embargo, a pesar de ser la escuela el contexto idóneo y de contarse con la voluntad política, en las mismas el trabajo educativo no ha logrado el necesario compromiso en las nuevas generaciones. Se hace necesario educar las relaciones con los ambientes, la consideración del carácter cualitativo de los mismos, y por tanto el uso de metodologías cualitativas que desentrañen las necesidades más acuciantes de las personas, grupos y comunidades y actuar en correspondencia con ellas. Esto con independencia de que la satisfacción de las mismas exija de tecnología de avanzada adecuada al problema, que, aunque no se cuente con los recursos, sí se conozca por la población y en su solución se brinden medidas objetivas, válidas y fiables de toda una serie de indicadores de calidad, tanto de vida, como de ambiente.

\section{La Psicología Ambiental en Cuba}

Los primeros estudios de Psicología Ambiental en nuestro país comienzan a finales de los años noventa con un análisis teórico del desarrollo de esta ciencia que se ha desarrollado en los Estados Unidos y en el Occidente europeo. Se observa la existencia de diferentes enfoques teóricos, nacidos en contextos distintos, con enfoques y objetivos distintos, y con una comprensión diferente del ambiente. Ello condujo en nuestro país hacia la asimilación crítica de todo lo conocido, e intentar integrar lo más 
conveniente a los nuevos objetivos, culminando con una nueva propuesta teórica que parte del Enfoque Histórico Cultural, enfoque teórico que se considera que brinda una visión más integrada, holística y comprensiva de la relación ser humano-ambiente y del comportamiento humano ante los cambios urgentes de la sociedad contemporánea. A partir de la base dialéctica de esta perspectiva teórica se definió como objeto de estudio, la formación y desarrollo de la consciencia ambiental, no como instancia interna, abstracta y aislada de lo externo, sino como un sistema de vivencias, conocimientos y acciones ambientales que junto a otras realidades psicológicas, el individuo realiza, experimenta activamente en su relación con el medio ambiente social, relación que puede ser armónica o disarmónica dependiendo de su sostenibilidad. En este sentido, la consciencia ambiental, se estructura paulatina y conscientemente, sufre sus períodos de crisis, que transita de lo disarmónico a lo armónico, de la inconsciencia o lo desconocido, a la consciencia o conocido. Este proceso se realiza a partir de las vivencias que el individuo va experimentando, acumulando y actualizando a lo largo de su historia personal (Pérez, 2000). De este modo los seres humanos se van conformando una imagen multidimensional del ambiente que la escuela puede instruir, aunque sea el propio niño con su activismo psíquico, quien integra esta enseñanza.

Los investigadores de la Psicología Ambiental desde el enfoque histórico cultural han apreciado la complejidad del ambiente y lo han tomado como unidad perceptiva, difícil de fragmentar, por lo que se valora ampliamente la percepción, en interrelación dinámica con los restantes procesos psíquicos superiores. (Alea, 2005). Esta interrelación influye en el desarrollo del resto de los procesos y dimensiones de la consciencia ambiental, meta final de todos los trabajos. Para alcanzar esta meta en los trabajos comunitarios se han definido diferentes direcciones de trabajos entre las cuales se encuentran:

- Luchar por el lugar que le corresponde a esta ciencia en la formulación de políticas públicas sobre medio ambiente.

- Sugerir la elevación de la cultura de participación de toda la población, incluidos los decisores de políticas.

- Trabajar en la evaluación de necesidades individuales, grupales y comunitarias.

- Trabajar por la concientización de aspiraciones de grupos e individuos en tema de calidad de vida que posee la población.

- Sugerir la información necesaria y dosificada de aspectos de interés ambiental para cada comunidad.

- Promover la comunicación entre sectores por búsqueda de soluciones ambientales a nivel local para disminuir la pobreza y el deterioro ambiental.

- Proponer nuevas bases para la creación de participación ciudadana comprometida.

- Propiciar la iniciativa y expectativas ante los problemas ambientales locales, estimulando el interés y la confianza en la solución a corto plazo. 
Para el logro de esto, se pueden utilizar los proyectos de Educación Ambiental, los que pueden lograr el enriquecimiento (cambio) de la percepción ambiental, teniendo en cuenta la etapa del desarrollo cognoscitivo de los individuos. Basados en que la percepción ambiental se modifica con el tiempo, coincidimos con estudiosos como Marcén y Sorando (1993) que han demostrado que el concepto de contaminación permanece un tanto invariable, mientras que el concepto de entorno tiende a ampliarse y profundizarse. Mientras que en los años 80 se relacionaba el medio ambiente con la naturaleza solamente, en los 90 este concepto se ha expandido a "todo lo que nos rodea", incorporando lo nuevo construido y lo social en líneas generales. Sin embargo en encuestas realizadas en nuestro país, aun se identifica el medio ambiente con "lo verde", lo que nos confirma de alguna forma, que el modelo teórico que todavía subyace en la educación ambiental se orienta hacia una visión naturalística como expresan Benegas y Marcén, (1995). La incorporación de los aspectos sociales a las actuaciones de educación ambiental será cada vez más aceptada en la medida que se desarrolle la definición que a continuación se formula y que se corresponde con la orientación teórica que profesamos.

\section{La investigación psicológica ambiental}

Para hablar de medio ambiente a partir del Enfoque histórico cultural, es importante reconocer el concepto del cual partimos. Nos adherimos a la definición propuesta en la Estrategia Nacional de Educación Ambiental cubana (1997) porque aúna y destaca significados claves que no deben quedar fuera. Así, se considera que el medio ambiente es "un sistema complejo y dinámico de interrelaciones ecológicas, socioeconómicas y culturales, que evoluciona a través del proceso histórico de la sociedad, abarca la naturaleza, la sociedad, el patrimonio histórico cultural, lo creado por la humanidad, la propia humanidad, y como elemento de gran importancia las relaciones sociales y la cultura", definición con la que todos o casi todos estamos de acuerdo, ya que contempla los elementos más importante del entorno, del sistema naturaleza-sociedad, también los productos sociales de la interacción humana con su entorno que no se circunscriben al daño natural, depredador e inconsciente sobre él para satisfacer las necesidades humanas actuales, sino que incluye lo inmaterial, el legado simbólico y cultural que es capaz. Ello no significa que todos la manejemos igual, ya que en última instancia volvemos a tender a la interpretación cosmovisiva que le otorga el sujeto del conocimiento de la propia ciencia en la que nos formamos. Sin embargo hay una característica que es insustituible desde cualquier interpretación de los problemas ambientales que conozcamos, trabajemos y resolvamos y es el carácter holístico e interactivo, por demás cambiante, que posee nuestro objeto de estudio.

En nuestro país se han realizado investigaciones en este sentido. Uno de los primeros estudios que se desarrolló en el contexto escolar y su entorno fue el desarrollado por Ramos (2001) con tutoría de la Dra. Febles, M. En el mismo se utilizaron varios métodos y técnicas de diagnóstico de la Consciencia Ambiental. Para ello se investigaron diferentes procesos (percepción, conocimientos y actitudes ambientales), con un carácter diagnóstico, siempre con el fin de definir aquellos 
aspectos más deficientes en los niños y hacia los cuales la escuela debía realizar un trabajo educativo futuro.

Entre los resultados obtenidos está la afirmación de que el Enfoque Histórico Cultural en Psicología, permite un análisis eficaz del fenómeno consciencia ambiental para lo que es necesario remitirnos a los procesos psicológicos (percepción, conocimientos, actitudes ambientales, en este caso) y sus interrelaciones que, formando un complejo sistema, participan en su formación y desarrollo. Esta concepción dinámica y desarrolladora nos obliga a rechazar toda perspectiva lineal y estática en la interrelación individuo - entorno. Los escolares de la muestra de manera general presentan una concepción incompleta sobre el medio ambiente, debido en lo fundamental, a que no lo consideran en aspectos sociales, solo físico-naturales, se observan niveles moderados de conocimiento ambiental, lo que determina bajos niveles de desarrollo de la consciencia ambiental, susceptibles de mejorar, aunque de forma general también existen debilidades en el desarrollo de las actitudes y comportamientos y en la correspondencia entre ellos. Se obtuvo que de los procesos estudiados los que más afectan la consciencia ambiental, son el bajo conocimiento de lo que es medio ambiente y por tanto de la percepción de los problemas ambientales que poseen, por ejemplo de las relaciones interpersonales (atracción-rechazo en el seno del grupo) y contra los que no se encamina una acción conciente. Por otro lado la existencia de incongruencia entre actitudes y comportamientos ambientales, que también proceden del desconocimiento del concepto y de la percepción limitada de los problemas que los aquejan. Existen inconsistencias en la formación de la consciencia ambiental a este nivel de enseñanza, debido principalmente a dificultades en la preparación y comunicación educativa de los maestros para llevar a cabo esta labor y a la falta de un programa sistemático, motivante y lo realmente efectivo que fomente la formación de dicha consciencia. A partir de los resultados obtenidos se realizó una propuesta interventiva para fomentar el desarrollo de la consciencia ambiental de los escolares, dirigida a las deficiencias concretas que se detectaron y en la cual se tendrán en cuenta la experiencia emocional (vivencias) y la cualidad de reflexión de los niños fundamentalmente, que serán parámetros para nuevas actividades de transformación. Aunque se observó una posición comprometida del niño a actuar a favor del desarrollo del medio ambiente natural, se desconoce su naturaleza social. Se pudieron revelar los aún bajos niveles de conocimiento y las potencialidades que para éste conocimiento tienen los niños. Del estudio se concluyó que se debe ampliar la definición de medio ambiente, y con ello concebirlo solo como fuente o condición para satisfacer sus necesidades. Habría que despertar su interés en función de la relación única que existe entre el individuo y éste en determinada etapa de su desarrollo, como decía L.S. Vygotski. Para ello se deben contemplar también las actitudes internas de los niños que posibilitan que, en conjunto con las condiciones externas, éstos alcancen una interacción más armoniosa (superior) con el medio a través de la formación (o transformación) de nuevas estructuras o funciones (neoformaciones, como motivos, valores, etc.) que posibiliten dicho salto.

La tarea de la formación de la consciencia ambiental en niños en la escuela debe contar con el apoyo conciente de la familia, directores de escuela, maestros y comunidad en general, de "mejores espacios" dentro de los cuales hacer posible esta 
transformación que llama al sistema de relaciones en el cual crece y se desarrollan nuestros niños, sobre todo el aula. A los maestros les corresponde una mejor preparación medioambiental para que la educación ambiental se oriente en el logro de un entorno no tan deseable como lograble en nuestras condiciones.

Los procesos de diagnóstico y evaluación que se llevaron a cabo en este trabajo constituyeron un momento inicial en el estudio de la consciencia ambiental, por el cual nos percatamos de la complejidad del estudio de la misma y la necesidad por tanto de acudir para el estudio a unidades de análisis, que permitan dar cuenta del curso del proceso de su formación como lo es la percepción ambiental con sus dimensiones e indicadores. Investigadores de esta rama del conocimiento científico han apreciado la complejidad del ambiente y lo han tomado como unidad perceptiva, que no puede ser fragmentada, más bien estudiada por dimensiones que se conectan y lo caracterizan, unidad perceptiva que está en estrecha interrelación dinámica con los restantes procesos psíquicos superiores (Alea, 2005).

Investigaciones del Centro de Información, Gestión y Educación Ambiental de CITMA (Ministerio de Ciencia, Tecnología y Medio Ambiente de Cuba) y del Centro de Investigaciones Psicológicas y Sociológicas (1999) sobre percepción ambiental, arrojan como resultados la relación directamente proporcional entre la instrucción, y la percepción de autorresponsabilidad de los estudiantes con los problemas del entorno. Por último, y como tercera variable, tienen en cuenta las propias características del entorno físico real que se percibe.

Investigaciones realizadas en jóvenes universitarios cubanos critican al plan de estudios por no contemplar más profundamente la dimensión ambiental (Betancourt y Febles, 2009). Pero si bien es necesaria la formación ambiental de los estudiantes universitarios, ésta no es posible lograrla si no se concibe una formación ambiental previa y consciente del profesorado. Uno de los retos ineludibles de la educación es precisamente la necesidad de contribuir a formar y capacitar no sólo a jóvenes y niños, sino también a los gestores, planificadores y las personas que toman las decisiones, para que orienten sus valores y comportamientos hacia una relación armónica con la naturaleza (Novo, 2009). Esto es así por el importante rol que juega el educador en este sentido, ya que esta formación puede y debe aparecer como un eje transversal para todo el currículo por tanto el profesor debe tener una mentalidad abierta, interdisciplinaria ya que la problemática ambiental rebasa cualquier enfoque unidireccional y de trabajo en equipo. Es necesario un enfoque sistémico, donde los objetos de la realidad se representen como sistemas organizados, integrados y jerarquizados unos con otros (Martínez, 2012).

La introducción de la dimensión ambiental en el currículo requiere la reflexión profunda del profesor y de su práctica pedagógica, para reorganizar el proceso docente. El profesor debe ser capaz de marchar junto a sus alumnos en el proceso de construcción del saber ambiental, de interiorización y aproximación de saberes significativos, tanto naturales como sociales y de formación para la toma de decisiones responsables. Esto igual se aplica a las otras enseñanzas. Ocurre que es más notoria en la enseñanza universitaria. 
En el trabajo referido se proponen pasos concretos para la introducción de la dimensión en cada disciplina. Lo cierto es que la formación ambiental debe estimular al estudiante a aprender del mundo y no sobre el mundo, aprender cómo funciona, cómo son sus relaciones. Es decir, incorporarse consciente y activamente en el proceso, para conocer la dinámica de la naturaleza y de la sociedad, a fin de saber actuar de manera acorde con sus necesidades, y no simplemente memorizar información sobre aspectos puntuales del ambiente.

Por otra parte apoyarse en la combinación de aspectos retrospectivos y prospectivos. Retrospectivos en el sentido de recordar, de reconstruir el pasado: cómo se actuó entonces y que procesos o fenómenos positivos o negativos ocurrieron; cuáles fueron los resultados; qué marchó bien, o qué acciones no fueron exitosas. Prospectivos, en el sentido de orientarse a la construcción del futuro, de qué debemos hacer, del logro de la visión deseable que esperamos alcanzar. Este proceso formativo debe enfatizar y estimular el autoaprendizaje, cada alumno debe construir sus propios saberes y entrenarse en formas autónomas de estudio y de investigación. Además de mostrar la relación entre teoría-práctica y viceversa. Se trata de una acción complementaria a la anterior y constituye el punto de partida para alcanzar una mejor comprensión de los fenómenos y hechos ambientales, por lo tanto, cuanto más frecuente sea su uso, más útil y productivo será su efecto (Betancourt y Febles, 2009).

\section{Estudios recientes sobre percepción ambiental}

En la investigación de Peña (2011) se utilizaron las dimensiones que los adolescentes pueden percibir del medio ambiente, específicamente del Medio Ambiente Escolar. Estas dimensiones pueden tomarse de criterio para profundizar en el conocimiento del medio ambiente en otros niveles de enseñanza. Se observó el grado de desarrollo de la percepción ambiental, evidenciándose que en los adolescentes predomina la dimensión natural, luego descubren la material o artefactual y la relacional, haciendo especial énfasis en los vínculos interpersonales. Por último se integran a la percepción la dimensión cultural, comportamental, intrapersonal y en menor medida la cognoscitiva, según las autoras. Los primeros resultados pudieran encontrar su explicación en que la percepción humana aún está determinada, en gran medida, por la actitud antropocéntrica y tendencia depredadora de las acciones humanas que se inclinan a la utilización de las riquezas naturales y objetos materiales del medio ambiente para satisfacer sus necesidades.

Se obtuvo que el desarrollo de la percepción ambiental está indisolublemente ligado al desarrollo cognoscitivo de los individuos, y éste se desarrolla con la práctica, sin embargo en los adolescentes de la investigación citada, aparte de las deficiencias de la dimensión ambiental en los programas de la escuela, por la edad que poseen aún son limitadas las experiencias que tienen con su ambiente, por tanto, la dimensión cognoscitiva es de las últimas en observarse. En este caso la educación ambiental, bien sea dentro de los programas de las diferentes materias, o fuera del currículo, debe jugar un papel primordial en el cambio actitudinal de los sujetos, favoreciendo un crecimiento moral en función de la naturaleza (Benegas y Marcén, 1995) y la sociedad 
a la que pertenecen. Además la construcción de las estructuras cognitivas del propio sujeto se apoya en la interacción con el medio. Además, una parte sustancial del pensamiento lógico y de la consciencia moral tienen sus raíces en la acción: surgen como reflexión consciente de la práctica (Díaz-Aguado y Medrano, 1994; Vygotski, 1996; Peña, 2011).

Se ha visto que el desarrollo de los valores es principalmente un proceso social y se va forjando progresivamente en las personas. De hecho, las influencias sociales van moldeando el sistema de valores hasta que éste se consolida y aún así se modifica según nuevas modas, creencias, doctrinas, etc. Las actitudes y los valores son aprendidos en función de una reestructuración de las escalas previas, que suele ser dinámica en la medida en que nos enfrentamos a nuevos retos o situaciones que implican una toma de postura. Debido a esa reestructuración no todos, aún perteneciendo a un mismo grupo social, y habiendo consolidado un conjunto de comportamientos con el entorno con bastantes rasgos similares, no poseemos un sistema de valores idéntico (Benegas y Marcén, 1995). Y es que otras razones de orden escolar, cultural y familiar están interviniendo en la esfera moral de la personalidad y de una u otra forma se refleja en el comportamiento. Es por ello que la educación moral que implica la educación ambiental en la escuela, es vía esencial para el desarrollo de la percepción ambiental.

Estudios en el campo del desarrollo de la percepción ambiental han demostrado que existen diferencias en cuanto al optimismo con el que los adolescentes vislumbran el futuro del planeta y en cómo perciben este tema los jóvenes (Anderson, 2007). Por tanto parece aconsejable que los educadores, además de valorar los conocimientos previos del alumno, asuman la tarea de detectar cuáles son sus intereses de partida y sobre todo qué aspectos o temas despiertan en ellos una mayor motivación o curiosidad por implicarse de forma más directa y activa (Benegas y Marcén, 1995).

En otros trabajos con escolares se encontró que se podrían constatar cambios favorables en las actitudes ambientales del grupo de adolescentes y con esto, en su consciencia ambiental. Su éxito estuvo en que se modificaron actitudes en adolescentes, a partir de la introducción de contenidos medioambientales en su sistema cognitivo, es decir nuevos conocimientos ampliaron su percepción ambiental, como dimensiones de la consciencia ambiental. Se logró gran implicación personal, condicionante básica para el elemento afectivo de las actitudes y su disposición a actuar hacia la transformación de su entorno. Se evidenciaron gran criticidad, problematización, reflexión hacia las temáticas ambientales fundamentalmente en las adolescentes (Peña, 2011).

En cuestionarios aplicados a los adolescentes se facilitó medir la dimensión cognoscitiva de la percepción ambiental de los adolescentes, contribuyendo una caracterización de la percepción del Medio Ambiente Escolar de estos adolescentes, espacio inmediato desde el cual se debe impulsar el trabajo educativo ambiental en la escuela. En el trabajo se constata que existe relación entre la participación en talleres $\mathrm{y}$ el desarrollo de la dimensión cognoscitiva de la percepción ambiental de adolescentes lo que permite proponer la realización de talleres como una vía sistemática para ampliar los conocimientos de los estudiantes y lograr la 
sensibilización de ellos con respecto a la temática ambiental (Solano; Zaneti y Rodríguez, 2012).

No obstante, en las revisiones realizadas al respecto sobre la base del elemento que mediatiza la relación escuela-comunidad, se reconoce que:

- A escala internacional se identifican dos tendencias: una que analiza el vínculo escuela-comunidad, orientado a la familia y destacando la necesidad de reforzar el trabajo de la escuela, y otra encaminada a reincorporar la escuela a su medio.

- En el ámbito nacional solamente se encuentran experiencias de escuelas que han desarrollado algún trabajo con la comunidad, a fin de consolidar su labor educativa.

- A escala local son mucho más escasos los estudios en esta dirección, con aisladas experiencias que muestren resultados en la práctica (Martínez, 2004).

Como se aprecia, la escuela es la encargada por excelencia de transmitir el sistema de conocimientos y habilidades acumulados por la sociedad. Esto requiere de una ampliación en las políticas sociales de desarrollo de la comunidad, involucrando al resto de las instituciones sociales (formales, no formales e informales) para hacer más efectivo el trabajo educativo, pues si bien muchos estudiantes conocen algunos aspectos de la problemática ambiental no existe un cambio es sus actitudes con respecto al tema. Existen muchos factores de tipo conflictivo y competitivo que pueden conforman nuestras acciones y decisiones diarias que hacen que no exista una relación directa entre el conocimiento de los problemas ambientales y un comportamiento responsable con respecto a este (Jaén y Barbudo, 2010).

La Educación Ambiental en Cuba, según las exigencias actuales, demanda un mejoramiento de la labor educativa que se desarrolla por parte de las instituciones educativas, dirigido a integrar los procesos ambientales con los de carácter educativos y actitudinales mediante la implementación de estrategias, acciones educativas y programas, para favorecer el desarrollo de una actitud ambiental positiva en los sujetos (Martínez, 2004).

Como decíamos la mayor parte de los problemas ambientales están dados porque la persona no puede percibir la magnitud de estos problemas. Los problemas existen y aumentan, sin embargo no son percibidos en tanto no afecta la conducta inmediata de las personas, se hace necesaria una percepción adecuada para que se actúe en conformidad al problema. Una adecuada percepción del ambiente y de sus afectaciones contribuye al desarrollo de una consciencia ambiental. En este caso la escuela es un ámbito de sensibilización, una de las principales vías de formación de hábitos y actitudes en cuanto al entorno, es por eso que muchos de las investigaciones que abordan la temática de la percepción ambiental utilizan estudiantes como sujetos de estudio. Es por este motivo que las investigaciones en el marco de la psicología ambiental realizadas en sujetos insertados en los diferentes niveles de enseñanza diseñados para niños, adolescentes y jóvenes son puntos cruciales en el incremento de una consciencia ambiental para la sociedad del mañana. Conocer las características de sus percepciones en cada uno de los niveles de enseñanza, permitiría evidenciar si el 
trabajo de educación ambiental que realizan las escuelas, los medios de difusión y la familia es efectivo. De ser así esperaríamos observar una amplitud de conocimientos y el desarrollo de la percepción del entorno de estos estudiantes. Si este desarrollo no fuese evidente, un estudio de este tipo nos ayudaría a detectar las debilidades que se observan a cada nivel y proponer soluciones prácticas que tributen a la educación ambiental. Por ello dando continuidad a las citadas investigaciones, entre las más recientes realizadas en el contexto cubano se encuentra la realizada por Tserej y Febles (2013) donde se analizan las posibles debilidades en cuanto a percepción ambiental en cada nivel de enseñanza y cuales pudieran ser las vías más propicias para lograr un correcto desarrollo de esta percepción. En esta investigación se maneja el concepto de percepción ambiental definido por Peña (2011) en el cual se entiende por percepción ambiental "aquel proceso cognoscitivo holístico e integral, que tiene un carácter selectivo, histórico y social, es una función psicológica que permite el conocimiento del mundo y se produce en la interacción con éste, está además en constante cambio y necesita actualizarse sistemáticamente, esta actualización a través de la enseñanza apunta a su buen funcionamiento. Depende en gran medida de la actividad de las personas que median su relación con el mismo y se encuentra relacionada con el sistema de sentidos psicológicos y vivencias que poseen las personas de su Medio Ambiente". En esta investigación se tuvieron en cuenta como indicadores aquellos empleados en la investigación de Peña (2011).

En la investigación de Tserej y Febles (2013) se detectó que la mayor parte de todos los estudiantes encuestados presentan un nivel de desarrollo bajo o mediano en cuanto a su percepción del ambiente. Las dimensiones ambientales más abordadas en los conceptos ambientales fueron la material, la natural y la comportamental; mientras que las menos mencionadas fueron la intrapersonal y la cultural. En sentido general, las vías más efectivas en cuanto temas medio ambientales fueron: la televisión, la escuela y los libros; por el contrario resulta significativo el hecho de que padres y familiares parecen jugar un papel ínfimo en la educación ambiental en los niños, adolescentes y jóvenes. En cuanto al análisis por géneros en estudiantes de pre-universitarios se constató que ambos grupos, muchachas y muchachos, demostraron en las encuestas igual interés en la protección del entorno. Algunos estudiantes plantearon que la contaminación acústica era uno de los principales problemas en la ciudad, lo cual resulta de mucho interés ya que se ha probado el efecto negativo del ruido en los procesos psicológicos y educativos de los alumnos (Estrada-Rodríguez y Méndez, 2010). Además, los estudiantes fueron capaces de reflexionar sobre la influencia de los peligros ambientales en la vida dentro del planeta Tierra por lo cual planteaban que era indispensable su protección. En este aspecto se observó un desarrollo en cuanto a las respuestas a medida que se ascendía en cuanto a niveles de enseñanza. Los estudiantes mayores eran capaces de ejemplificar los nexos que existen entre la problemática ambiental y los trastornos de la humanidad. Sin embargo contradictoriamente la mayor parte de los estudiantes universitarios se clasificaron con bajo desarrollo de percepción ambiental ya que muchas de sus respuestas demostraron sino desconocimiento, apatía con respecto al tema. Esto pudiera estar relacionado con la insuficiencia en cuanto a la educación ambiental en los niveles anteriores, y aquella que proporcionan los profesores en esta carrera específicamente. 
Estudios llevados a cabo por la Universidad de Murcia en el año 2010 apuntan resultados similares en estudiantes españoles de enseñanza secundaria. Los autores plantean que la instrucción formal, no está incidiendo, en general, de forma patente en las creencias y actitudes hacia el medio ambiente en el estudiantado (Jaén y Barbudo, 2010). En América Latina se aprecia también un comportamiento impropio del estudiantado con respecto a la dimensión ambiental (Silva y García, 2010). Independientemente de la diferencias culturales, sociales y económicas de nuestros países la inefectividad de la educación ambiental persiste como un problema común que debe ser estudiado y comprendido por los diferentes gestores y planificadores de los programas de estudio en los diferentes sistemas de enseñanza; con el objetivo de incluir la temática ambiental de modo transversal e interdisciplinario en cada uno de los currículos.

\section{Conclusiones}

En las investigaciones psicológicas ambientales de Cuba se constata que el contexto más idóneo y vía principal para la educación ambiental es la escuela. La psicología ambiental como base para el trabajo educativo es aún incipiente. La percepción es una de las dimensiones más importantes de la consciencia ambiental que es factible de desarrollar a través de acciones que utilizan la vivencia y la reflexión de los estudiantes. Al desarrollarse la percepción se amplían y enriquecen otros procesos psicológicos por la vía de los vínculos que establecen los sistemas psicológicos. Estos vínculos los mantienen interrelacionados genética, estructural y funcionalmente. El desarrollo de la percepción ambiental está indisolublemente ligado al desarrollo cognoscitivo y afectivo de los individuos, y ésta se desarrolla con la práctica, de manera que los seres humanos se van conformando una imagen multidimensional del ambiente con el que interactúa, la escuela puede instruir, aunque sea el propio niño con su activismo psíquico, quien integra esta enseñanza. La escuela, dígase la enseñanza en la escuela, debe estimular al estudiante a aprender del mundo y no sobre el mundo, aprender cómo funciona, cómo son sus relaciones.

En investigaciones realizadas en Cuba, la tarea de la formación de la consciencia ambiental en niños en la escuela debe contar con el apoyo consiente de la familia, directores de escuela, maestros y comunidad en general, contar con "mejores espacios" dentro de los cuales se hace posible esta transformación que llama al sistema de relaciones en el cual crece y se desarrollan nuestros niños, sobre todo el aula. Se han detectado aspectos que hay que vencer en el trabajo en la escuela, en que a pesar de los esfuerzos que se realizan en esta dirección, aún subsisten insuficiencias que tienen innumerables manifestaciones en la escuela. Ellas se vinculan con:

- La falta de un accionar coherente y sistemático que integre los esfuerzos de las instituciones educativas en la protección del Medio Ambiente Comunitario.

- Inadecuado tratamiento de la problemática ambiental con un carácter integral por parte de las instituciones educativas de la comunidad.

- El tratamiento parcial, y en ocasiones aislado, de los aspectos relacionados con los 
conocimientos, la percepción y la sensibilidad de la población ante los problemas del Medio Ambiente Comunitario.

- La concepción didáctico-metodológica para el tratamiento de los problemas ambientales de la comunidad a partir de un estudio de diagnóstico integral.

- Un enfoque disperso y parcializado para dirigir el proceso de evaluación de la Educación Ambiental de la Comunidad.

- La insuficiente utilización de indicadores para evaluar la situación real del Medio Ambiente comunitario de una manera integrada.

\section{Referencias bibliográficas}

ALEA, G. A. (2005). Diagnóstico y potenciación de la Educación Ambiental en jóvenes universitarios. Tesis de Maestría en Desarrollo Social Caribeño. Ciudad de La Habana: Facultad Latinoamericana de Ciencias Sociales.

AMÉRIGO, M. (2006). La investigación en España sobre actitudes proambientales y comportamiento ecológico. Medio Ambiente y comportamiento humano,7 (2), 4571.

ANDERSON, I. K. (2007). Junior Secondary School Pupils' Perception of the Relevance of Environmental Issues: Implications for Science Educators. African Journal of Educational Studies in Mathematics and Sciences, 5, 87-98.

BENEGAS, J. y MARCÉN, C. (1995). La educación ambiental como desencadenante del cambio de actitudes ambientales. Revista complutense de educación, 6 (2), 1128.

BETANCOURT, A. y FEBLES, M. (2009). Acerca de la introducción de la dimensión ambiental en la educación universitaria. Ciudad de La Habana: Reduniv.

DÍAZ-AGUADO, M. J. y MEDRANO, C. (1994). Educación y razonamiento moral. Una aproximación constructivista para trabajar los contenidos transversales. Bilbao: Mensajero.

ESTRADA-RODRÍGUEZ, C. y MÉDEZ, I. (2010). Impacto del ruido ambiental en estudiantes de educación primaria de la Ciudad de México. Revista Latinoamericana de Medicina Conductual, 1 (1), 57-68.

FEBLES, M. (2011). Cultura y Educación Ambiental. Ciudad de La Habana: Centro de Estudios del Medio Ambiente

GONZÁLEZ, A. y AMÉRIGO, M. (1999). Actitudes hacia el medio ambiente y conducta ecológica. Psicothema, 11 (1), 13-25.

JAÉN, M. y BARBUDO, P. (2010). Evolución de las percepciones medioambientales de los alumnos de educación secundaria en un curso académico. Revista Eureka sobre Enseñanza y Divulgación de las Ciencias, 7 (número extraordinario), 247259. 
MARCÉN, C. y SORANDO, M. (1993). Cómo conciben, perciben y valoran su entorno los escolares zaragozanos. Investigación en la Escuela, 20, 65-80.

MARTíNEZ, C. M. (2004). La educación ambiental para el desarrollo del trabajo comunitario en las instituciones educativas. Tesis doctoral en Ciencias Pedagógicas. Holguín: Instituto pedagógico de Holguín.

MARTÍNEZ, R. (2012). Ensayo crítico sobre educación ambiental. Diálogos educativos, 12 (24), 70-104.

MUÑOZ, J. M. (2007). La pedagogía de los espacios como discurso de la educación ambiental. Bordon, 59 (4), 641-657.

NOVO, M. (2009). La educación ambiental, una genuina educación para el desarrollo sostenible. Revista de Educación, número extraordinario, 195-217.

CUBA. MINISTERIO DE CIENCIA TECNOLOGÍA Y MEDIO AMBIENTE (1997). Estrategia Nacional de Educación Ambiental. Ciudad de La Habana: Centro de Información Divulgación y Educación Ambiental (CIDEA).

PEÑA, Y. (2011). "Actuando...percibirás". Una propuesta de procedimiento metodológico grupal para la caracterización de la percepción del medio ambiente escolar. Tesis de Diploma en Psicología. Ciudad de La Habana: Facultad de Psicología.

PÉREZ, D. (2000). Hacia Una Psicología Ambiental en Cuba. Tesis de Diploma en Psicología. Ciudad de La Habana: Facultad de Psicología.

RAMOS, M. (2001) Psicología Ambiental: Un estudio de la conciencia Ambiental en el entorno escolar. Tesis de Diploma en Psicología. Ciudad de La Habana: Facultad de Psicología

RODRÍGUEZ, S. (1986). Percepción Ambiental. En Jiménez, F. y Aragonés, J. I. (Ed.) Introducción a la Psicología Ambiental (pp. 53-63). Madrid: Editorial Alianza.

SILVA, O. y GARCÍA M. (2010). La dimensión ambiental en el comportamiento cotidiano del estudiante de la tercera etapa de educación básica. Educare, 14 (2), 7 30 .

SOLANO, O. ZANETI, P. y RODRÍGUEZ, R. (2012). El desarrollo de la percepción ambiental escolar en un grupo de adolescentes de la ESBU Felipe Poey Aloy. La Habana: Facultad de Psicología.

STEVENS, P. (2010). Embedment in the environment: A new paradigm for wellbeing? Perspectives in Public Health, 130 (6), 265-269.

TSEREJ, O. y Febles, M. (2013). Análisis del grado de desarrollo en la percepción ambiental en estudiantes de diferentes niveles de enseñanza. Eco-solar, 40, Consultada el 21 de junio de 2013, http://www.cubasolar.cu/biblioteca/ecosolar.htm

VYGOTSKI, S.L. (1996). Obras Escogidas. Madrid: Editorial Visor.

YUS, R. (1994). Dos mundos contradictorios. Cuadernos de Pedagogía, 227, 22-27. 


\section{Correspondencia con las autoras}

Olga Natalia TSEREJ VÁZQUEZ

Museo Nacional de Historia Natural de Cuba

Obispo 61, Plaza de Armas, Habana Vieja, Habana, Cuba CP 10100

Teléfono: (537) 8632589

e-mail: tserej@gmail.com

\section{María M. FEBLES ELEJALDE}

Facultad de Psicología de la Universidad de La Habana

San Rafael 1168 esquina Mazón, Plaza de la Revolución, La Habana, C.P 10400

Teléfono: (537) 8704617

e-mail: mariaf@rect.uh.cu 